\title{
Imaging Study on Acupuncture Inhibiting Inflammation and Bone Destruction in Knee Osteoarthritis Induced by Monosodium lodoacetate in Rat Model
}

\author{
Qian $\operatorname{Tan}\left(\mathbb{D}^{1, *}\right.$ \\ Zhengkun Cai $\mathbb{1}^{2, *}$ \\ Jia $\mathrm{Li} \mathbb{D D}^{3}$ \\ Jing $\mathrm{Li}^{1}$ \\ Hongchun Xiang (1D \\ Bocun $\mathrm{Li}^{\prime}$ \\ Guowei Cai' \\ 'Department of Acupuncture, Union \\ Hospital, Tongji Medical College, \\ Huazhong University of Science and \\ Technology, Wuhan, People's Republic of \\ China; ${ }^{2}$ Department of Public \\ Administration, Huazhong University of \\ Science and Technology, Wuhan, People's \\ Republic of China; ${ }^{3}$ College of \\ Acupuncture and Orthopedics, Hubei \\ University of Chinese Medicine, Wuhan, \\ People's Republic of China \\ *These authors contributed equally to \\ this work
}

Correspondence: Guowei Cai Department of Acupuncture, Union Hospital, Tongji Medical College, Huazhong University of Science and Technology, Wuhan, People's Republic of China

Email cgw645@163.com
Objective: We aim to explore whether acupuncture inhibits inflammation and bone destruction in rat model monosodium iodoacetate (MIA)-induced knee osteoarthritis (KOA) by 18Ffluorodeoxyglucose (18F-FDG) small-animal positron emission tomography (PET) and micro-computed tomography (CT) imaging.

Methods: KOA was induced in rats by intra-articular injection MIA $(2 \mathrm{mg} / 50 \mu \mathrm{L})$ through the right knee of the rats. Forty male Sprague Dawley rats weighing 280 to $340 \mathrm{~g}$ (12 weeks old) were randomly divided into four groups including Control group, KOA group, KOA plus manual acupuncture group (KOA+MA), KOA plus sham acupuncture group (KOA $+\mathrm{SA}$ ). The acupuncture treatment lasted for three weeks (one-day rest after six days of treatment). Paw withdrawal threshold test and open-field test were used to assess mechanical allodynia and locomotor activity respectively for once a week. Hematoxylin and eosin (H\&E) staining was used to assess the damage of the cartilage, synovium and infrapatellar fat pad (IFP). 18F-FDG PET was performed to quantify joint inflammation. The influence on the subchondral bone in these rats was confirmed by micro-CT.

Results: Mechanical hyperalgesia, joint inflammation, and obvious bone destruction were observed in the KOA group. H\&E staining of the knee joint found that manual acupuncture played a protective effect in cartilage, synovium and IFP destruction. However, compared with KOA group, the results in sham acupuncture had no significant difference. After manual acupuncture treatment in KOA rats, inflammation was significantly suppressed shown by 18F-FDG PET imaging. Micro-CT analysis of the knee joint revealed that manual acupuncture protected bone by inhibiting osteophyte development and subchondral bone remodeling. Conclusion: The results of $18 \mathrm{~F}-\mathrm{FDG}$ PET and micro-CT showed that manual acupuncture inhibited inflammation and bone destruction, which provides reliable evidence for the effectiveness of acupuncture in hindering development of KOA, and provides reliable evidence for clinical application of acupuncture.

Keywords: osteoarthritis, acupuncture, PET, micro-CT, inflammation, bone destruction

\section{Introduction}

Osteoarthritis (OA), a common skeletal and muscle disease, is one of the main causes of global disability. ${ }^{1}$ The number of patients with the prevalence and the incidence increase with age has increased to more than 240 million worldwide. ${ }^{2}$ While the knee joint is the most common site affected in $\mathrm{OA}^{3}$ The clinical manifestations of knee osteoarthritis (KOA) are joint pain, swelling, stiffness and 
even dyskinesia, ${ }^{4,5}$ which affects the quality of life of patients and aggravates the medical burden of society. ${ }^{6}$ Variety of physiological and mechanical factors affect the occurrence and development of KOA, involving age, sex, obesity, heredity trauma, and mechanical stress. ${ }^{7}$

The characteristic case changes all tissues in knee of KOA include articular and meniscal cartilage degeneration and loss, subchondral bone changes, synovial joint inflammation ${ }^{8}$ and infrapatellar fat pad (IFP) inflammation and fibrosis. ${ }^{9}$ Inflammation is strongly implicated in the pathogenesis of $\mathrm{KOA},{ }^{10}$ because $\mathrm{KOA}$ is correlated with aging mechanisms such as the presence of an inflammatory microenvironment and the impaired link between inflammasomes and autophagy. ${ }^{11}$ Previous studies ${ }^{12-15}$ have reported that inflammation develops within the joint structures, the formation of which is associated with the activation of numerous immune cells which secrete cytokines amplifying the inflammatory process, including tumor necrosis factor alpha (TNF- $\alpha$ ) and interleukin (IL), such as IL-1 1 .IL-6, IL-8 and so on. One study ${ }^{16}$ suggested that conditioned media from all tissues of OA patients produced high levels of IL-6, IL-8, and CCL2. CCL21, MMP-3, which means all tissues of joint participate in inflammatory process. Meanwhile, There is increasing evidence $^{17}$ that subchondral bone plays an important role in the progression of $\mathrm{OA}$ in recent years. Recent studies ${ }^{18,19}$ showed that there are distinct microstructural alterations in subchondral bone at different stages of OA. Bone homeostasis is maintained by a balance between bone remodeling, osteoblastic bone formation, and osteoclastic bone resorption. ${ }^{20}$ Once subchondral bone is destroyed, bone homeostasis would be broken, which is involved in the pathophysiology of OA at the biochemical and mechanical levels. Intra-articular injection of monosodium iodoacetate (MIA) is a chemical method to induce $\mathrm{KOA}^{21}$ which inhibits the activity of glyceraldehyde 3-phosphate dehydrogenase in chondrocytes, resulting in the destruction of glycolytic pathway. ${ }^{22}$ Because the site of injection is restricted to the joint space, intra-articular injection of MIA causes mainly chondrocyte cell death, leading to cartilage degeneration and subsequent subchondral bone alterations. ${ }^{23}$ Furthermore, this model is widely used for pain research and efficacy evaluation of therapeutic interventions. ${ }^{24}$

At present, the etiology of KOA remains unclear and there is no radical cure for KOA. ${ }^{25}$ The aim of treatment is mainly limited to relieving knee pain, enhancing mobility and function. Acupuncture is one of the most popular treatments applied in traditional Chinese medicine, which has been generally applied to relieve pain in musculoskeletal diseases. ${ }^{26}$ In recent years, acupuncture has been widely used to treat KOA, and its effectiveness and safety have been abundantly proved in clinical and experimental studies. ${ }^{27,28}$ Despite being mature technology, the exact mechanism of acupuncture is still unclear. Meanwhile, few researchers aim to observe the therapeutic effect of the KOA animal model through imaging analysis. In this study, our aim was to investigate the effectiveness of acupuncture in the treatment of KOA from the perspective of imaging. Therefore, positron emission tomography (PET) and microcomputed tomography (CT) will be used to investigate the protective effects of acupuncture on inflammation and subchondral bone in KOA rats in our study.

\section{Materials and Methods}

\section{Animals and Model Induction}

Studies were conducted in accordance with the Chinese Guidelines of Animal Care and Welfare. Approval was granted by the Animal Care and Use Committee of Hubei University of Chinese medicine (Wuhan, China) for all animal procedures. For all animal experiments, the experimenters assessing the outcomes were blinded to the intervention. Forty male Sprague Dawley rats weighing 280 to $340 \mathrm{~g}$ (12 weeks old) were group housed in a room with controlled temperature $\left(22 \pm 2^{\circ} \mathrm{C}\right)$ under a reversed 12-hour light/dark cycle, and the rats had free access to food and water. The rats were divided into four groups by random number method: Control group (Control, $\mathrm{n}=10)$, KOA group (KOA, $\mathrm{n}=10$ ), KOA plus manual acupuncture group (KOA+MA, $\mathrm{n}=10)$, KOA plus sham acupuncture group $(\mathrm{KOA}+\mathrm{SA}, \mathrm{n}=10)$. Rats were briefly anesthetized with isoflurane and received either intra-articular injection through the infrapatellar ligament of the right knee with monosodium iodoacetate (MIA; $2 \mathrm{mg} / 50 \mu \mathrm{L}$, in saline, KOA group) or saline $(50 \mu \mathrm{L}$, Control group) using a 27-gauge, 0.5 -inch needle.

\section{Acupuncture Treatment}

One week after intra-articular injection of MIA, the rats of $\mathrm{KOA}+\mathrm{MA}$ group were treated with $0.30 \mathrm{~mm} \times 13 \mathrm{~mm}$ acupuncture needles, which were inserted into an acupoint on the right with a depth of $5 \mathrm{~mm}$. The acupoint named "Zusanli" (ST36), located at $2 \mathrm{~mm}$ lateral to the anterior tubercle of the tibia and $5 \mathrm{~mm}$ below the capitulum fibulae under the knee joint in the rats. The needles were frequently 
manual rotated for one minute every five minutes. This procedure is repeated for six times (total time is 30 minutes) during the manipulation session. Stimulation was performed once daily for a period of three weeks (one-day rest after six days of treatment). For KOA+SA group, sham acupoint (1$2 \mathrm{~cm}$ lateral to the ST36 for rat) was selected in ipsilateral side. The time of operations and penetration depth in sham acupuncture were the same as the manual acupuncture group, but needles were not manually twisted in sham acupuncture. These treatments were performed without anesthesia. The experimental timeline was shown in Figure 1.

\section{Positron Emission Tomography (PET) Imaging}

No rat death was observed during the experiment. After the last treatment, four rats in each group were randomly selected for PET imaging. The rats were fasted overnight before the PET scan. Anesthetized by inhalation of $2 \%$ isoflurane, rats were injected with $500 \pm 25 \mu \mathrm{Ci} 18 \mathrm{~F}-\mathrm{FDG}$ (Wuhan Union Hospital PET Center) through the tail vein. After 60 minutes of FDG uptake, the anesthetized rats were fixed on the scanning bed for 21-minute micro-PET scan by the Trans-PET Bio Caliburn 700 system (Raycan Technology Co., Ltd, Suzhou, China). The PET images were reconstructed using the three-dimensional (3D) OSEM (iteration number was 2 , subset number was 12) method with a voxel size of $0.5 \times 0.5 \times 0.5 \mathrm{~mm}^{3}$. Gaussian image post-filtering can be applied with the following parameters: none, low (window size $=3$, sigma $=1$ ).
Images were displayed with software Carimas (Turku PET Center, Turku, Finland), AMIDE (Amide's a Medical Imaging Data Examiner) and Pmod (Pmod Technologies LLC, Switzerland). The mean standardized uptake value (SUV) was calculated using the following formula: mean pixel value with the decay-corrected region-of-interest activity $(\mu \mathrm{Ci} / \mathrm{kg}) /($ injected dose $[\mu \mathrm{Ci}] /$ weight $[\mathrm{kg}])$.

\section{Micro-computed Tomography (CT) Imaging}

The rats were euthanized with $\mathrm{CO}_{2}$, and the right knee joint of the rats was dissected and fixed in $4 \%$ paraformaldehyde (PFA). Micro-CT was used to evaluate the tibial subchondral bone in the rat specimens. A Bruker SKYSCAN 1276 scanner micro-CT system (Bruker, Karlsruhe, Germany) was used to image the subchondral bone regions in rats at a voltage of $85 \mathrm{kV}$ and a current of $200 \mu \mathrm{A}$ to obtain images having a pixel size of $10 \mu \mathrm{m}$ and using $1 \mathrm{~mm} \mathrm{Al}$ filter to capture the best X-ray projections, with the platform software being used to assess three-dimensional (3D) morphometric analyses, density, and other parameters. We reconstructed and analyzed the 3D structure and morphometry in a doubleblind manner. The area between the proximal tibia and the distal femur was selected for analysis of the following parameters: trabecular bone volume fraction $(\mathrm{BV} /$ TV), trabecular spacing (Tb.Sp), trabecular number (Tb.N), and trabecular thickness (Tb.Th).

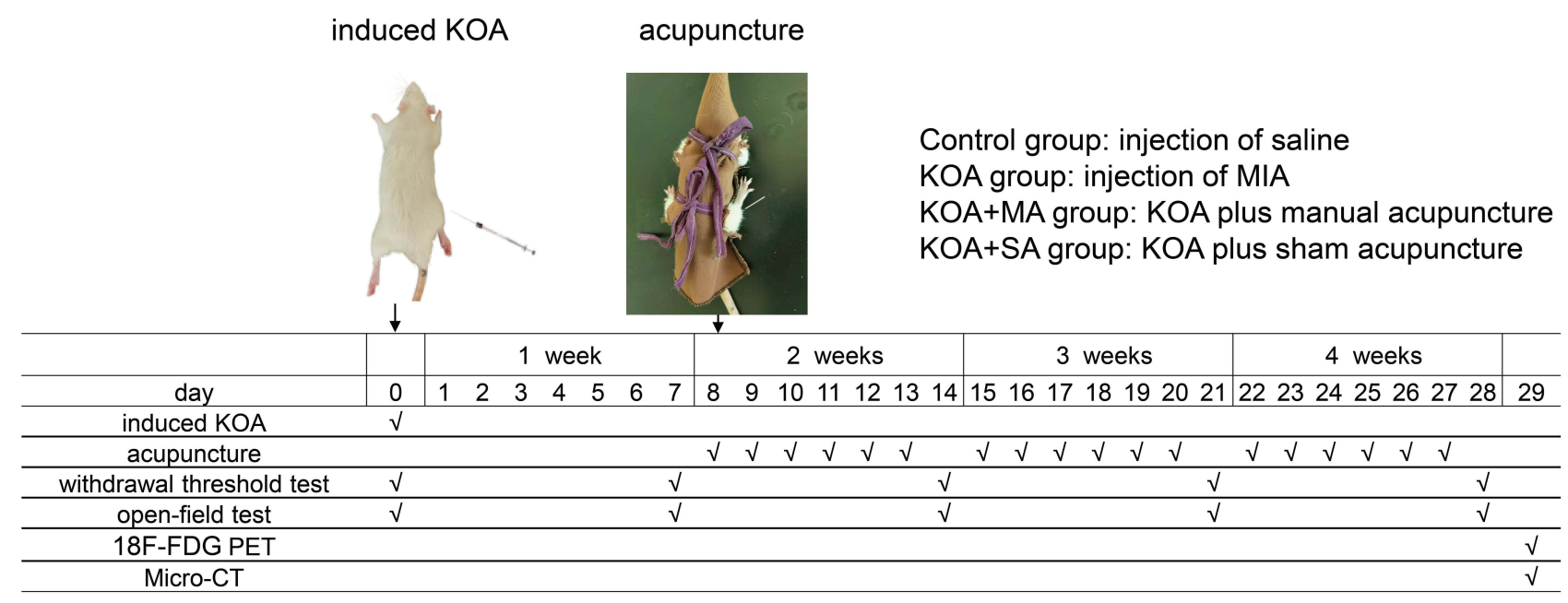

Figure I Experiment timeline. KOA was induced in rats by intra-articular injection MIA ( $2 \mathrm{mg} / 50 \mu \mathrm{L})$ through their right knee, while the rats in the Control group were injected with saline at the same position. Rats in experimental groups were underwent following treatments: manual acupuncture or sham acupuncture treatment was performed at the seventh day after modeling and lasted for three weeks. Nociceptive behavioral tests were measured once a week until the treatment was completed. After the last treatment, four rats in each group were randomly selected for I8F-FDG PET imaging. Micro-CT were operated after the rats were sacrificed. 


\section{Histopathological Analysis}

Following micro-CT scanning, tissues were fixed with $4 \%$ PFA, decalcified in $10 \%$ EDTA, embedded in paraffin. Then sagittal sections with a thickness of $5 \mu \mathrm{m}$ were prepared. Histopathological change of knee joint was observed under an optical microscope after hematoxylin and eosin (H\&E) staining. Synovial hyperplasia, inflammatory infiltration, and infrapatellar fat pad (IFP) fibrosis were analyzed by H\&E staining, including cartilage, synovium and infrapatellar fat pad. Meanwhile, according to the Mankin scoring standards ${ }^{29}$ (Table S1), the pathology the cartilage was calculated. Synovial inflammation was assessed by histopathological synovitis score according to the status of tissue architecture and pathological change in H\&E staining ${ }^{30}$ (Table S2).

\section{Nociceptive Behavioral Tests}

Severity of joint pain was quantitatively evaluated by paw withdrawal threshold using Electric Von Frey (IITC Life Science Inc., CA, USA). Rats were individually placed in metal cages, which allowed full access to the paws, and the rats were acclimated at least 30 minutes before the test. After the rats were quiet, the probe was applied perpendicularly the mid-plantar surface of the right hind paw to evaluate the withdrawal threshold. Until the force elicited a nocifensive response (ie, paw lifting, jumping), data was recorded on the display screen. This procedure was repeated three times with an interstimulus interval of three minutes. The mean values of the three tests were used for statistical analysis.

\section{Locomotor Activity Test}

Open-field test was used to evaluate the alterations in locomotor activity. Prior to testing all rats were transferred to the testing room followed by a 30-minute acclimatization period. The rats were placed at the center of a $1 \times 1 \times 0.5 \mathrm{~m}$ chamber and allowed free exploration for five minutes. During this period, the locomotor activity of the rats was recorded using a digital camera placed above the open field apparatus which was connected to a computer. The computer would save and measure activity data, including total distance, average speed, and rest time. The measurement frequency was once a week.

\section{Statistical Analysis}

GraphPad Prism (version 8.3.0; GraphPad Software Inc., San Diego, CA, USA) was applied to statistical analysis.
The results were expressed as mean \pm standard deviation (SD). Comparisons between different groups were analyzed by one-way analysis of variance (ANOVA) with Dunnett's multiple comparison test or two-way ANOVA with Tukey's multiple comparison test with repeated measures, followed by post hoc Šidák's multiple comparison tests. Bonferroni post hoc tests were used for multiple comparisons. $P<0.05$ was considered to be statistically significant.

\section{Results \\ MA Inhibits the Joint Inflammation in KOA Rats}

PET was used to show the changes of glucose metabolism, which indicated the degree of joint inflammation ${ }^{31} \mathrm{We}$ obtained transverse, sagittal and coronal view of $18 \mathrm{~F}-$ FDG PET scan of rat right knee joints (Figure 2A). All the ROIs (region of interest) assessed in the right knees of KOA group, comparing to the Control group presented with significantly higher FDG uptake quantified by SUVmax $(P<0.01)$. Compared to the KOA group, the rats of the KOA+MA group showed lower uptake of tracer $(P<0.05)$. The difference of SUVmax between the KOA group and $\mathrm{KOA}+\mathrm{SA}$ group did not reach statistical significance $(P>0.05)$ (Figure 2B).

\section{MA Inhibits the Joint Bone Destruction in KOA Rats}

Micro-CT was used to quantify the structural changes in the tibial subchondral bone in these rats. Reconstructed 3D micro-CT images of knee joints in each group were shown in Figure 3A and B, typical 3D reconstructed micro-CT images of the proximal tibial metaphyseal trabecular bone in different groups. Compared with the Control group, the scan results showed that the KOA group and $\mathrm{KOA}+\mathrm{SA}$ group exhibited obvious bone destruction, which was manifested as an increase in tibial subchondral osteolysis. Meanwhile, the number and connection density of subchondral bone trabeculae decreased, and the bone trabeculae were sparse and disorderly distributed in the KOA group. The bone destruction phenomenon was significantly improved after treatment with manual acupuncture. Statistically, the values of BV/TV, Tb.N and Tb.Th of the model group were downregulated $(P<0.01)$, and the values of $\mathrm{Tb} . \mathrm{Sp}$ were upregulated than the Control group $(P<0.01)$. Meanwhile, compared with the model group, the manual acupuncture group prominently increased the 


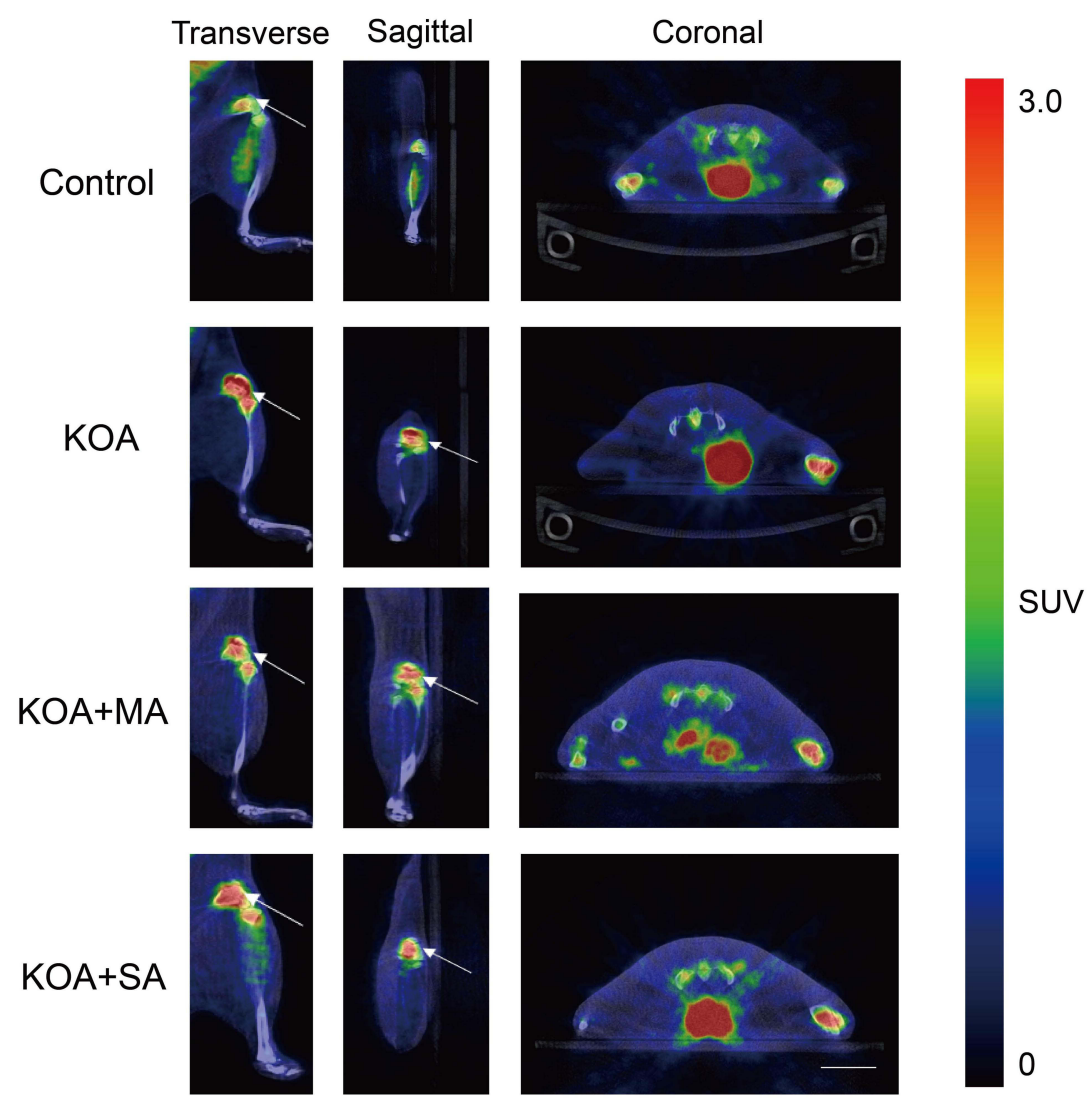

A

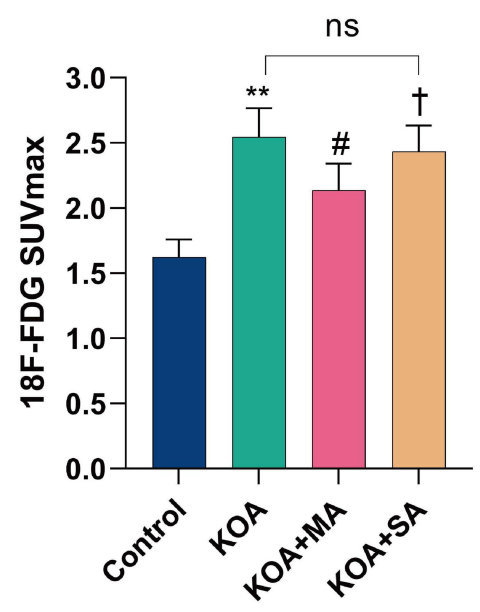

B

Figure 2 Effect of MA on inhibiting inflammation in KOA rats. (A) I8F-FDG PET imaging of mouse right knee joints from transverse, sagittal, and coronal views. Inflammatory site (white arrow). (B) Region of interest (ROIs) I8F-FDG SUVmax from each group. Data are presented as the mean \pm standard deviation, $\mathrm{n}=4$ /group; Twoway ANOVA was used. KOA group vs Control group ( ${ }^{* * P<0.0 I) ; ~ K O A+M A ~ g r o u p ~ v s ~ K O A ~ g r o u p ~}\left({ }^{\#} P<0.05\right)$; KOA+MA group vs KOA+SA group $\left({ }^{\dagger} P<0.05\right)$, KOA+SA group vs KOA group (no significant difference. $P>0.05$ ).

values of $\mathrm{BV} / \mathrm{TV}$, Tb.N and Tb.Th $(P<0.01)$, while the value of Tb.Sp showed the opposite results $(P<0.01)$. However, the parameter analysis of rat knee micro-CT between the KOA group and the $\mathrm{KOA}+\mathrm{SA}$ group had no significant difference $(P>0.05)$.

\section{MA Inhibits Cartilage, Synovium and IFP Destruction in KOA Rats}

Gross microscopy was used to observe the damage of the articular cartilage in the knee joint of rats after treatment. H\&E staining was applied to assess the histopathological change of cartilage, synovium and IFP. The keen joint in $\mathrm{KOA}$ group and $\mathrm{KOA}+\mathrm{SA}$ group showed severe cartilage injury, while manual acupuncture significantly alleviated injury (Figure 4A). There was no histopathological variation in the joint of normal rats which showed normal histological structure of the cartilaginous articular surface and synovial membrane, but significant degradation of the cartilage layer and inflammatory cells infiltration as well as congested blood vessels were demonstrated in the knee joint of KOA group. Cartilage destruction was prominently alleviated in the $\mathrm{KOA}+\mathrm{MA}$ group, while cartilage destruction of the rats did not ameliorate in the $\mathrm{KOA}+\mathrm{SA}$ group (Figure 4B). The results of Mankin scores in each group were shown in Figure 4E. $\mathrm{KOA}+\mathrm{MA}$ group was significantly lower than the KOA group and $\mathrm{KOA}+\mathrm{SA}$ group, while the KOA group and $\mathrm{KOA}+\mathrm{SA}$ group had no significant difference, which reflected the change of cartilage tissue. Meanwhile, compared with the Control group and $\mathrm{KOA}+\mathrm{MA}$ group, synovial cell layer was thicker and the cell density of the matrix increased in the $\mathrm{KOA}$ group and $\mathrm{KOA}+\mathrm{SA}$ group (Figure 4C). The synovitis score results were consistent with the above results (Figure 4F). The hyperplastic changes in the IFP surface and the fibrosis of IFP were showen in Figure 4D in the $\mathrm{KOA}$ group and $\mathrm{KOA}+\mathrm{SA}$ group. However, the IFP destruction was significantly improved after the treatment 
Control

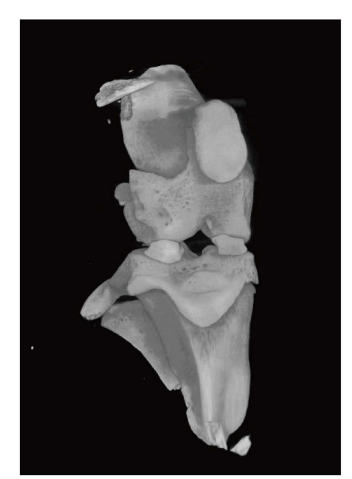

B
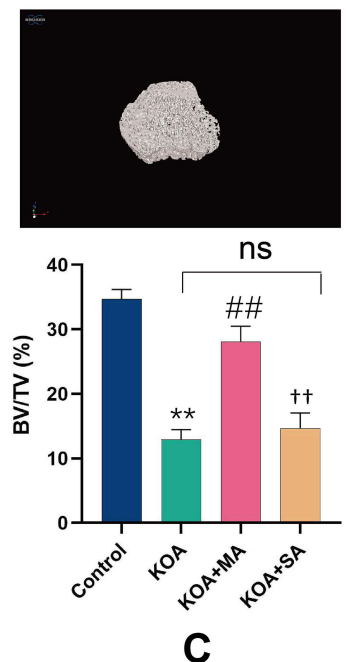

KOA
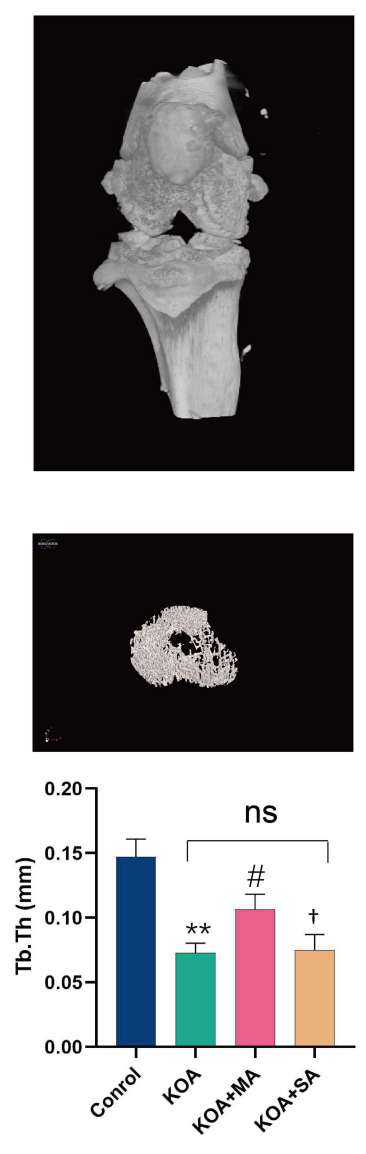

D
$\mathrm{KOA}+\mathrm{MA}$
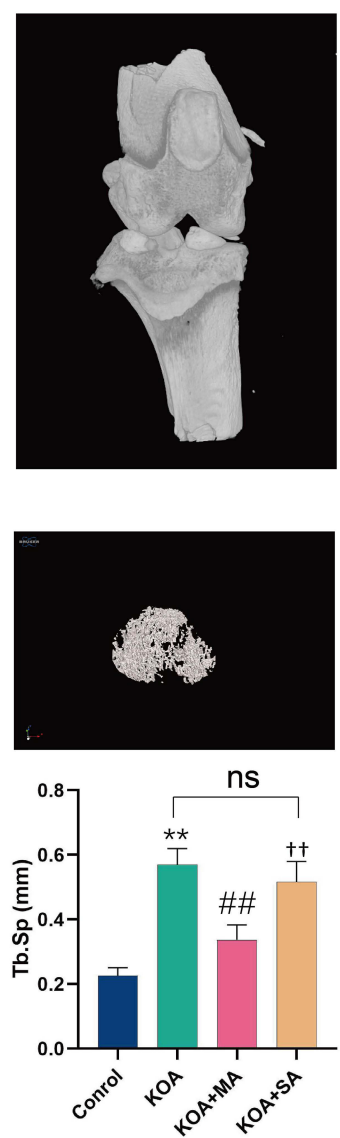

E
$\mathrm{KOA}+\mathrm{SA}$
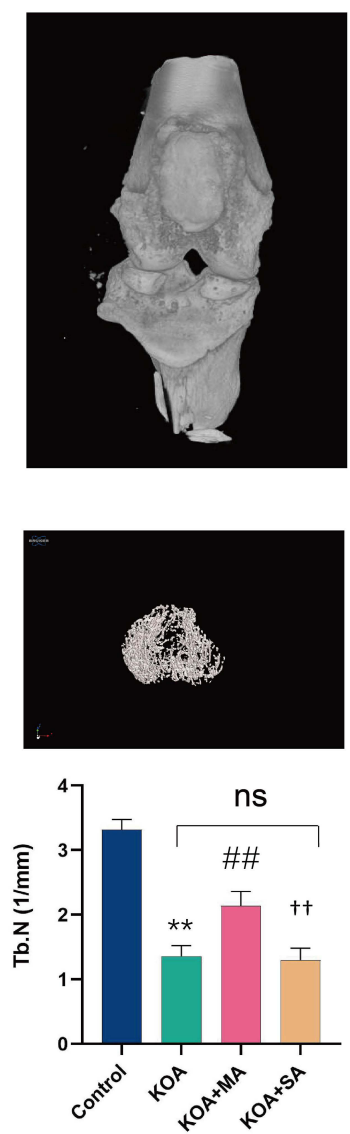

$\mathbf{F}$

Figure 3 Effect of MA on inhibiting the joint bone destruction in KOA rats. (A) Reconstructed 3D micro-CT images of knee joints in each group. (B) 3D reconstruction of tibial plateau trabecular bone in different group. (C) The values of BV/TV in each group. (D) The values of Tb.Th in each group. (E) The values of Tb.Sp in each group. (F) The values of Tb.N in each group. Data are presented as the mean \pm standard deviation, $n=6 /$ group; KOA group vs Control group (**P<0.0I); KOA+MA group vs KOA group $\left({ }^{\#} P<0.05,{ }^{\#} P<0.0 I\right)$; KOA+MA group vs KOA+SA group $\left({ }^{\dagger} P<0.05,{ }^{\dagger} P<0.01\right)$, KOA+SA group vs KOA group (no significant difference, $P>0.05$ ).

Abbreviations: BV/TV, bone volume fraction; Tb.Th, trabecular thickness; Tb.Sp, trabecular spacing; Tb.N, trabecular number.

with manual acupuncture. These results suggested that manual acupuncture could alleviate the damage of cartilage, synovium and IFP in KOA.

\section{MA Alleviates Mechanical Allodynia and Improves Locomotor Activity in KOA \\ Rats}

We tested the nociceptive behavior which was induced by intra-articular injection of MIA into the right knee of rats. Prior to the start of the MA treatment, IA injection of MIA significantly decreased paw withdrawal threshold (PWT) in ipsilateral, comparing to intra-articular injection of saline (Control group). Compared with the KOA group and KOA + SA group, manual acupuncture significantly attenuated pain behavior of $\mathrm{KOA}(\mathrm{KOA}+\mathrm{MA})$ at two weeks to four weeks.
However, there were no differences between the magnitudes of these measures between the KOA group and $\mathrm{KOA}+\mathrm{SA}$ group (Figure 5A).

The open field test was used to detect the spontaneous activity of the rats. The timeline is the same as above (Figure 1). The results including total distance, average speed, and rest duration of rats to assess the mobility, and the motion trail were recorded. Compared with the Control group, MIA meaningfully reduced the pain thresholds and spontaneous activity of KOA mice in the first week. In the second week to fourth week, compared with the KOA group or $\mathrm{KOA}+\mathrm{SA}$ group, $\mathrm{MA}$ treatment significantly increased total distance (Figure 5B) and average speed (Figure 5C) $(P<0.01)$, while it significantly reduced rest duration (Figure 5D) $(P<0.01)$. 


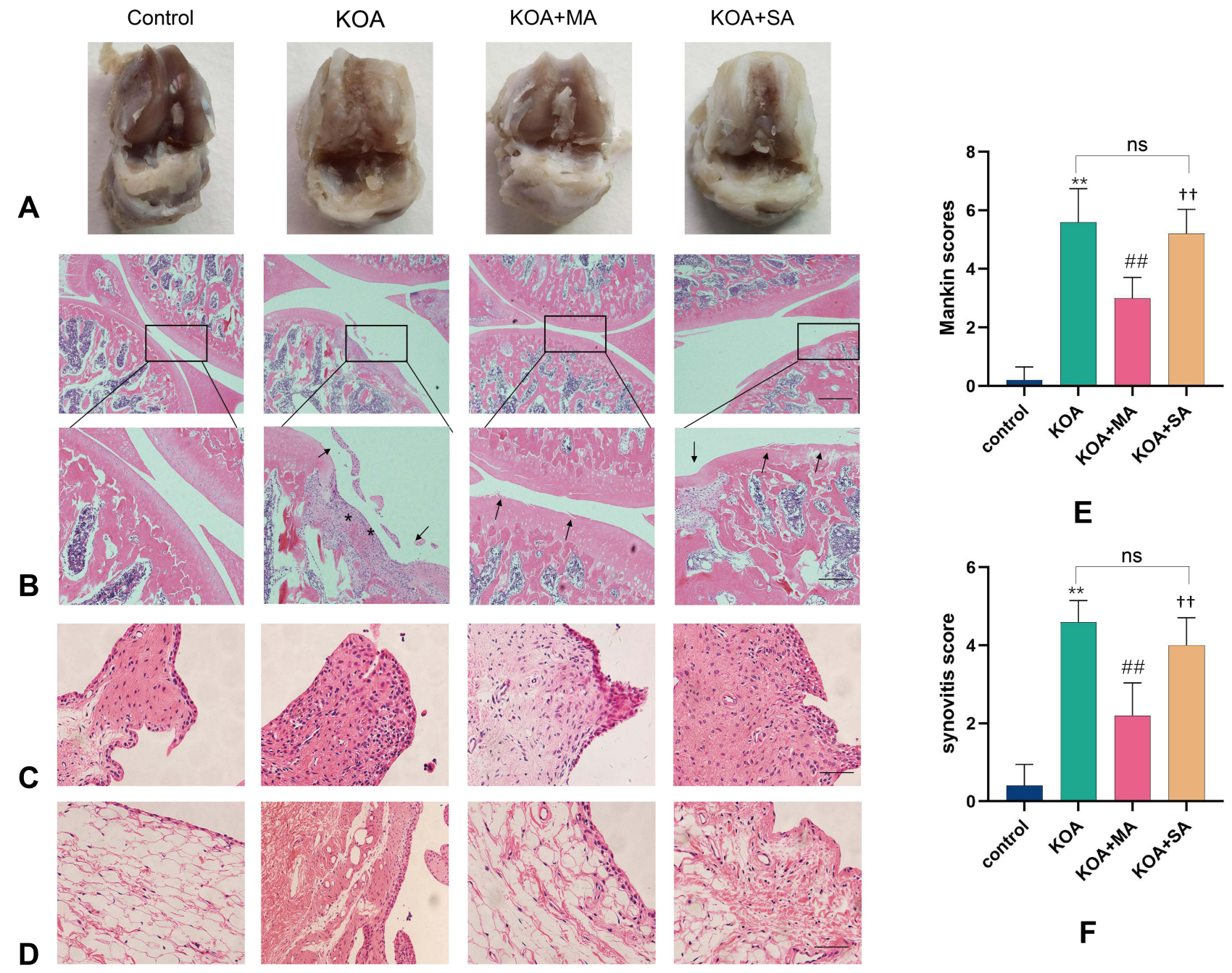

Figure 4 Effect of MA on protecting cartilage, synovium and IFP damage in KOA rats (A) Macroscopic view of articular cartilage damage surface in each group. (B) H\&E staining (original magnification, $40 \times$ and $100 \times$ ), synovial hyperplasia, inflammatory cell infiltration (*), cartilage surface damage (black arrow). (C) Synovial inflammation. 200×. (D) Fibrosis of IFP. 200×. (E) Mankin scores in each group. (F) Synovitis score. Data was presented as the mean \pm standard deviation, $\mathrm{n}=5$ /group; KOA group vs Control group (**P<0.0I), KOA+MA group vs KOA group ( $\left.{ }^{\# \#} P<0.01\right)$, KOA+MA group vs KOA+SA group ( $\left.{ }^{\dagger \dagger} P<0.0 \mathrm{I}\right)$.

Therefore, these results demonstrated that manual acupuncture had remarkable inhibitory effect on pain and improved activity ability.

\section{Discussion}

In this study, 18F-FDG PET and micro-CT imaging were applied to explore the effect of acupuncture on rat model of MIA-induced KOA, and whether acupuncture inhibits inflammation and bone destruction in the development of KOA. We used 18F-FDG PET to quantify effectively the inflammatory activity of KOA and its therapeutic response on manual acupuncture. In our study, the results of PET suggested that manual acupuncture significantly decreased the glucose uptake, suggesting that MA inhibited local inflammatory reaction of KOA. Therefore, the results of
H\&E staining further confirmed this conclusion that manual acupuncture not only reduced cartilage injury, but also reduced synovial inflammation and fibrosis of IFP. In our study, the results show that the number, volume, and thickness of trabeculae were significantly increased, and the distance between trabeculae was reduced after treatment with manual acupuncture, compared with KOA rats, which exhibited that acupuncture could inhibit severe bone erosion. Consequently, we considered that acupuncture had a protective effect on subchondral bone for KOA. Meanwhile, we identified that acupuncture showed an obvious effect on analgesia, and the results were consistent with our previous experiments ${ }^{32}$ that we demonstrated manual acupuncture reduced hyperalgesia and provides a new explanation on the mechanism of acupuncture in 

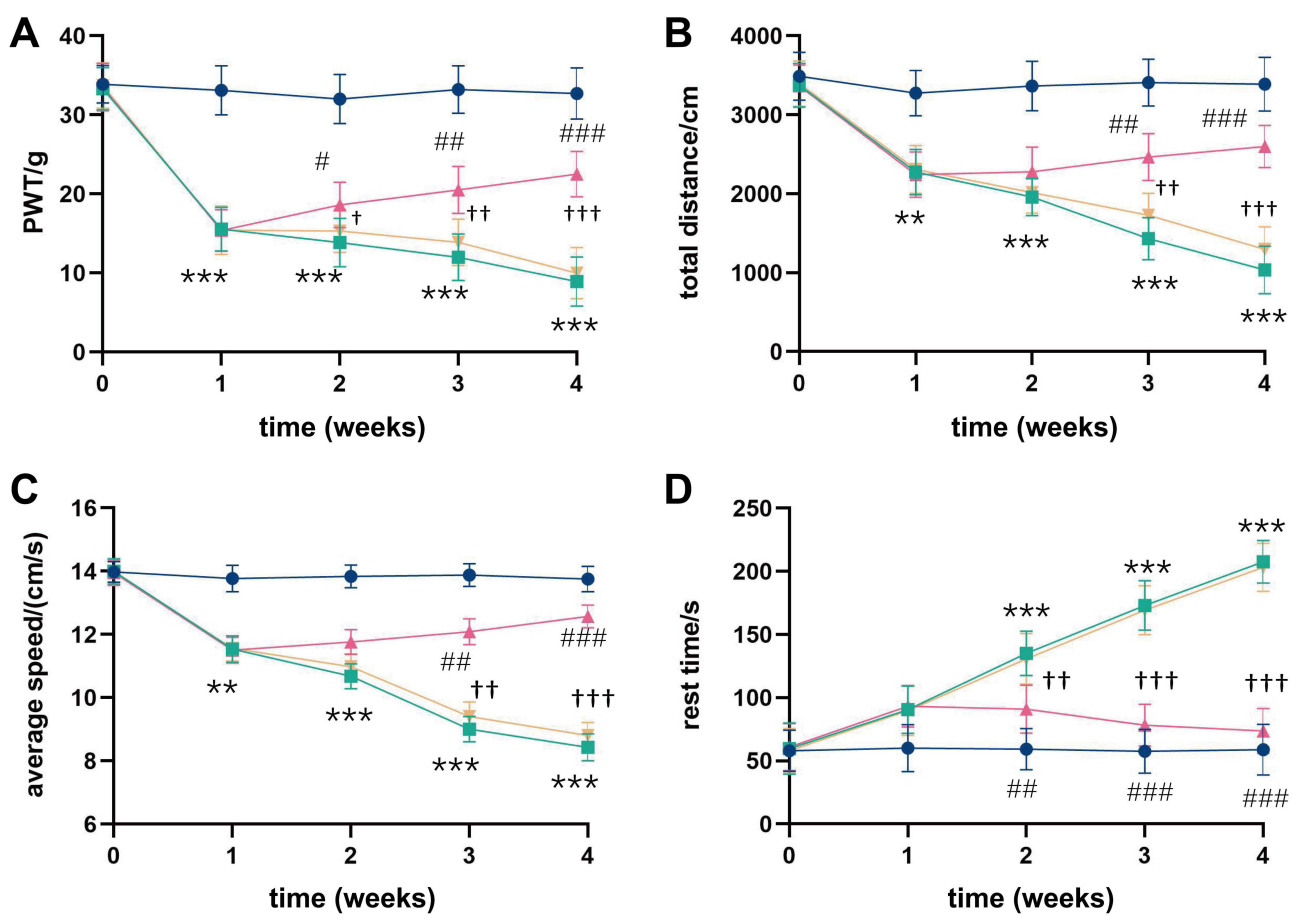

- Control $\quad-$ - KOA

$\triangle \mathrm{KOA}+\mathrm{MA}$

$\mathrm{KOA}+\mathrm{SA}$

Figure 5 Effect of MA on alleviating mechanical allodynia and improving locomotor activity in KOA rats. (A) PWT was tested by Electric Von Frey before modeling and measured every week. (B-D) represented total distance, average speed and rest time respectively. Two-way ANOVA was used in different time periods. Data was presented

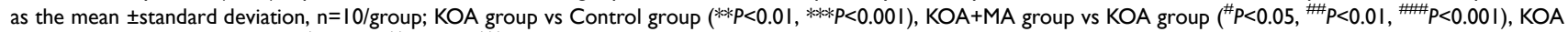
+ MA group vs KOA+SA group $\left({ }^{\dagger} P<0.05,{ }^{\dagger \dagger} P<0.01,{ }^{+\dagger \dagger} P<0.00 I\right)$.

treating KOA. In a recent study, Hoshino et al found that synovial inflammation and destruction of subchondral bone was observed as soon as seven days after injection in high-dose MIA $(1.0 \mathrm{mg} / 30 \mu \mathrm{L}) .{ }^{33}$ Consequently, we used an MIA-induced KOA model to observe the therapeutic effects of MA on pain, inflammation and motor function in KOA model rats.

In the current study, the diagnosis of KOA is based on clinical and imaging findings. ${ }^{34}$ Although the gold standard for OA imaging is conventional radiography in daily clinical practice, it has a few limitations, such as low sensitivity, compared to $\mathrm{PET} / \mathrm{CT},{ }^{35}$ which is relatively expensive but enables us to obtain more information. ${ }^{36}$ 18F-FDG PET/CT imaging is usually applied to detect the glucose uptake and metabolic changes in human diseases $^{37}$ using 18F-fluorodeoxyglucose (18F-FDG), a radioactively labelled glucose analogue, which is injected into the body to reflect the status of metabolism activities through the aggregation of radioactivity. ${ }^{38,39}$ The 18F-FDG uptake was associated with serological and clinical markers of inflammation, ${ }^{40}$ and the role of $18 \mathrm{~F}-\mathrm{FDG}$ PET in imaging inflammatory conditions has been validated in $\mathrm{KOA}^{41}$ The high expression of GLUT-1 receptor subtype in stimulated inflammatory cells such as macrophages is one of the potential mechanisms for $18 \mathrm{~F}$ FDG uptake in these inflammatory conditions. ${ }^{41}$ Meanwhile, pro-inflammatory factors obviously increases high metabolic activity of activated inflammatory cells with high glucose uptake and glycolysis process, leading to avid FDG uptake in inflammatory tissues. ${ }^{42}$ However, the $18 \mathrm{~F}-\mathrm{FDG}$ as a glucose analogue cannot be further metabolized after phosphorylation, which accumulates within cells, then we can quantify $18 \mathrm{~F}-\mathrm{FDG}$ uptake at sites of pathological increased glucose metabolism also in the musculoskeletal tissue. ${ }^{31}$ Therefore, 18 F-FDG accumulation in the knee suggests that inflammatory cytokines enhanced inflammatory activity.

In recent years, micro-CT has become the gold standard for evaluating the microstructure of subchondral bone and trabecular morphological of bone plate in small animal models, such as rats and mice. ${ }^{43}$ Three-dimensional structure of bone trabecula by micron scanning reconstruction, can accurately measure the overall bone mass parameters and a large number of bone structure parameters of the 
specimen. $^{44}$ The imaging and data indicate osteophyte development and subchondral bone remodeling, which could be used to observe bone destruction in the development of the KOA.

Acupuncture has been accepted in China's Guidelines for the Diagnosis and Treatment of Osteoarthritis ${ }^{45}$ and the Guidelines of the American Academy of Orthopedic Surgeons. ${ }^{46}$ Compared with sham acupuncture, effective acupuncture is to penetrate into acupoints with a certain depth and give a certain degree of stimulation. There are many clinical and animal studies on acupuncture in the treatment of KOA, which confirm that acupuncture indeed improves the symptoms of KOA. ${ }^{27,47}$ Previous studies have found that acupuncture reduces inflammation and pain by regulating a variety of pathways, ${ }^{48,49}$ such as MCP2/CCR2 signaling pathway and nuclear factor- $\kappa \mathrm{B}$ signaling pathway, but few studies investigated acupuncture in reducing the degree of inflammation and protecting subchondral bone from the perspective of imaging. Therefore, our results confirmed that the effect of manual acupuncture was significantly better than sham acupuncture in inhibiting inflammation and bone destruction during the procedure in KOA by PET imaging and micro-CT.

However, limitations have to be noted in this study. We only detected PET and micro-CT imaging after treatment, and did not observe the dynamic changes during treatment, and did not give insight into even longer longitudinal changes after treatment. Furthermore, we have not studied its molecular mechanism in our study. Further studies are needed to explore the effect of acupuncture on KOA during treatment and the local metabolic changes of the joint for a long time after treatment, and what molecular mechanisms are involved in these changes.

In summary, our research shows that manual acupuncture could inhibit inflammation and bone destruction by 18F-FDG PET and micro-CT. And the results provide a new theoretical basis for acupuncture in the treatment of KOA.

\section{Abbreviations}

KOA, knee osteoarthritis; MIA, monosodium iodoacetate; 18F-FDG, 18F-fluorodeoxyglucose; PET, positron emission tomography; micro-CT, micro-computed tomography; IFP, infrapatellar fat pad; BV/TV, Bone volume fraction; Tb.Th, trabecular thickness; Tb.Sp, trabecular spacing; Tb. N, Trabecular number; PWT, paw withdrawal threshold.

\section{Funding}

The authors disclosed receipt of the following financial support for the research, authorship, and/or publication of this article: This work was supported by the Natural Science Foundation of China (No: 81774410), and the Project of Hubei Provincial Health Committee (No: ZY2021M018).

\section{Disclosure}

The authors report no conflicts of interest in this work.

\section{References}

1. Katz JN, Arant KR, Loeser RF. Diagnosis and treatment of hip and knee osteoarthritis: a review. JAMA. 2021;325(6):568-578. doi:10.1001/jama.2020.22171

2. Cross M, Smith E, Hoy D, et al. The global burden of hip and knee osteoarthritis: estimates from the global burden of disease 2010 study. Ann Rheum Dis. 2014;73(7):1323-1330. doi:10.1136/annrheumdis2013-204763

3. Roberts NL, Mountjoy-Venning WC, Anjomshoa M. Global, regional, and national incidence, prevalence, and years lived with disability for 310 diseases and injuries, 1990-2015: a systematic analysis for the Global Burden of Disease Study 2015. Lancet. 2016;388 (10053):1545-1602. doi:10.1016/S0140-6736(16)31678-6

4. Butterfield NC, Curry KF, Steinberg J, et al. Accelerating functional gene discovery in osteoarthritis. Nat Commun. 2021;12(1):467. doi:10.1038/s41467-020-20761-5

5. Kidd B. Mechanisms of pain in osteoarthritis. HSS j. 2012;8 (1):26-28. doi:10.1007/s11420-011-9263-7

6. Glyn-Jones S, Palmer AJ, Agricola R, et al. Osteoarthritis. Lancet. 2015;386(9991):376-387. doi:10.1016/S0140-6736(14)60802-3

7. Hunter DJ, Bierma-Zeinstra S. Osteoarthritis. Lancet. 2019;393 (10182):1745-1759. doi:10.1016/S0140-6736(19)30417-9

8. Roseti L, Desando G, Cavallo C, Petretta M, Grigolo B. Articular cartilage regeneration in osteoarthritis. Cells. 2019;8(11):1305. doi:10.3390/cells 8111305

9. Kouroupis D, Willman MA, Best TM, Kaplan LD, Correa D. Infrapatellar fat pad-derived mesenchymal stem cell-based spheroids enhance their therapeutic efficacy to reverse synovitis and fat pad fibrosis. Stem Cell Res Ther. 2021;12(1):44.

10. Li L, Li Z, Li Y, Hu X, Zhang Y, Fan P. Profiling of inflammatory mediators in the synovial fluid related to pain in knee osteoarthritis. BMC Musculoskelet Disord. 2020;21(1):99. doi:10.1186/s12891-0203120-0

11. Rezuș E, Cardoneanu A, Burlui A, et al. The link between inflammaging and degenerative joint diseases. Int J Mol Sci. 2019;20(3):614. doi:10.3390/ijms20030614

12. Singh M, Valecha S, Khinda R, et al. Multifactorial landscape parses to reveal a predictive model for knee osteoarthritis. Int J Environ Res Public Health. 2021;18(11):5933. doi:10.3390/ijerph18115933

13. Loukov D, Karampatos S, Maly MR, Bowdish DME. Monocyte activation is elevated in women with knee-osteoarthritis and associated with inflammation, BMI and pain. Osteoarthritis Cartilage. 2018;26(2):255-263. doi:10.1016/j.joca.2017.10.018

14. Mun CJ, Letzen JE, Nance S, et al. Sex differences in interleukin-6 responses over time following laboratory pain testing among patients with knee osteoarthritis. j Pain. 2020;21(5-6):731-741. doi:10.1016/ j.jpain.2019.11.003

15. Chow YY, Chin KY. The role of inflammation in the pathogenesis of osteoarthritis. Mediators Inflamm. 2020;2020:8293921. doi:10.1155/ 2020/8293921 
16. Belluzzi E, Olivotto E, Toso G, et al. Conditioned media from human osteoarthritic synovium induces inflammation in a synoviocyte cell line. Connect Tissue Res. 2019;60(2):136-145. doi:10.1080/ 03008207.2018.1470167

17. Hu W, Chen Y, Dou C, Dong S. Microenvironment in subchondral bone: predominant regulator for the treatment of osteoarthritis. Ann Rheum Dis. 2020;80(4):413-422. doi:10.1136/annrheumdis-2020218089

18. Hügle T, Geurts J. What drives osteoarthritis?-synovial versus subchondral bone pathology. Rheumatology. 2017;56(9):1461-1471. doi:10.1093/rheumatology/kew389

19. Bettica P, Cline G, Hart DJ, Meyer J, Spector TD. Evidence for increased bone resorption in patients with progressive knee osteoarthritis: longitudinal results from the Chingford study. Arthritis Rheum. 2002;46(12):3178-3184. doi:10.1002/art.10630

20. Tanaka Y, Okada Y, Nakamura T. Inter- and intracellular signaling in secondary osteoporosis. J Bone Miner Metab. 2003;21(2):61-66. doi: $10.1007 / \mathrm{s} 007740300010$

21. de Sousa Valente J. The pharmacology of pain associated with the monoiodoacetate model of osteoarthritis. Front Pharmacol. 2019;10:974. doi:10.3389/fphar.2019.00974

22. Muley MM, Krustev E, Reid AR, McDougall JJ. Prophylactic inhibition of neutrophil elastase prevents the development of chronic neuropathic pain in osteoarthritic mice. J Neuroinflammation. 2017;14 (1):168. doi:10.1186/s12974-017-0944-0

23. Bryk M, Chwastek J, Mlost J, Kostrzewa M, Starowicz K. Sodium monoiodoacetate dose-dependent changes in matrix metalloproteinases and inflammatory components as prognostic factors for the progression of osteoarthritis. Front Pharmacol. 2021;12:643605. doi:10.3389/fphar.2021.643605

24. Lampropoulou-Adamidou K, Lelovas P, Karadimas EV, et al. Useful animal models for the research of osteoarthritis. Eur j Orthopaedic Surg Traumatol. 2014;24(3):263-271. doi:10.1007/s00590-013-1205-2

25. Ou L, Meng Y, Chen Z, et al. Evidence of Chinese herbal fumigation for knee osteoarthritis: a protocol for systematic review and meta-analysis. Medicine. 2021;100(6):e24532. doi:10.1097/ MD. 0000000000024532

26. Lin ML, Wu JH, Lin CW, et al. Clinical effects of laser acupuncture plus Chinese cupping on the pain and plasma cortisol levels in patients with chronic nonspecific lower back pain: a randomized controlled trial. Evid Based Complementary Alternative Med. 2017;2017:3140403. doi:10.1155/2017/3140403

27. Tu JF, Yang JW, Shi GX, et al. Efficacy of Intensive Acupuncture Versus Sham Acupuncture in Knee Osteoarthritis: a Randomized Controlled Trial. Arthritis Rheumatol. 2021;73(3):448-458. doi:10.1002/art.41584

28. Hunter DJ, Harris RE. Acupuncture and knee osteoarthritis: does dose matter? Arthritis Rheumatol. 2021;73(3):371-373. doi:10.1002/art.41583

29. Sahin K, Kucuk O, Orhan C, et al. Niacinamide and undenatured type II collagen modulates the inflammatory response in rats with monoiodoacetate-induced osteoarthritis. Sci Rep. 2021;11(1):14724. doi:10.1038/s41598-021-94142-3

30. Krenn V, Morawietz L, Burmester GR, et al. Synovitis score: discrimination between chronic low-grade and high-grade synovitis. Histopathology. 2006;49(4):358-364. doi:10.1111/j.13652559.2006.02508.x

31. Menendez MI, Hettlich B, Wei L, Knopp MV. Preclinical Multimodal Molecular Imaging Using (18) F-FDGPET/CT and MRI in a Phase I Study of a Knee Osteoarthritis in In Vivo Canine Model. Mol Imaging. 2017;16:1536012117697443. doi:10.1177/ 1536012117697443

32. Luo Z, Hu Z, Bian Y, et al. Scutellarin Attenuates the IL-1 $\beta$-induced inflammation in mouse chondrocytes and prevents osteoarthritic progression. Front Pharmacol. 2020;11:107. doi:10.3389/fphar.2020. 00107
33. Hoshino T, Tsuji K, Onuma H, et al. Persistent synovial inflammation plays important roles in persistent pain development in the rat knee before cartilage degradation reaches the subchondral bone. BMC Musculoskelet Disord. 2018;19(1):291. doi:10.1186/s12891-018-2221-5

34. Feng L, Feng C, Wang CX, et al. Circulating microRNA let-7e is decreased in knee osteoarthritis, accompanied by elevated apoptosis and reduced autophagy. Int $J$ Mol Med. 2020;45(5):1464-1476. doi:10.3892/ijmm.2020.4534

35. Peters M, de Jong J, Scharmga A, et al. An automated algorithm for the detection of cortical interruptions and its underlying loss of trabecular bone; a reproducibility study. BMC Med Imaging. 2018;18(1):13. doi:10.1186/s12880-018-0255-7

36. Hayashi D, Roemer FW, Guermazi A. Imaging of Osteoarthritis by Conventional Radiography, MR Imaging, PET-Computed Tomography, and PET-MR Imaging. PET Clin. 2019;14(1):17-29. doi:10.1016/j.cpet.2018.08.004

37. Vaidyanathan S, Patel CN, Scarsbrook AF, Chowdhury FU. FDG $\mathrm{PET} / \mathrm{CT}$ in infection and inflammation-current and emerging clinical applications. Clin Radiol. 2015;70(7):787-800. doi:10.1016/j. crad.2015.03.010

38. Hess S, Hansson SH, Pedersen KT, Basu S, Høilund-Carlsen PF. FDG-PET/CT in Infectious and Inflammatory Diseases. PET Clin. 2014;9(4):497-519, vi-vii. doi:10.1016/j.cpet.2014.07.002

39. Yin WY, Yuan J, Zhang ZM, et al. (18)F-fluorodeoxyglucose positron emission tomography-computed tomography for assessing organ distribution of stressed red blood cells in mice. Sci Rep. 2021;11 (1):2505. doi:10.1038/s41598-021-82100-y

40. Agca R, Blanken AB, van Sijl AM, et al. Arterial wall inflammation is increased in rheumatoid arthritis compared with osteoarthritis, as a marker of early atherosclerosis. Rheumatology. 2021;60 (7):3360-3368. doi:10.1093/rheumatology/keaa789

41. Saboury B, Parsons MA, Moghbel M, et al. Quantification of aging effects upon global knee inflammation by 18F-FDG-PET. Nucl Med Commun. 2016;37(3):254-258. doi:10.1097/MNM.0000000000000430

42. Min Z, Amlani M. Pulmonary Mycobacterium kansasii Infection Mimicking Malignancy on the (18) F-FDGPET Scan in a Patient Receiving Etanercept: a Case Report and Literature Review. Case Rep Pulmonol. 2014;2014:973573. doi:10.1155/2014/973573

43. Bouxsein ML, Boyd SK, Christiansen BA, Guldberg RE, Jepsen KJ, Müller R. Guidelines for assessment of bone microstructure in rodents using micro-computed tomography. J Bone Mineral Res. 2010;25(7):1468-1486. doi:10.1002/jbmr.141

44. Song Y, Zhu F, Lin F, Zhang F, Zhang S. Bone quality, and the combination and penetration of cement-bone interface: a comparative micro-CT study of osteoarthritis and rheumatoid arthritis. Medicine. 2018;97(35): e11987. doi:10.1097/MD.0000000000011987

45. Zhang Z, Huang C, Jiang Q, et al. Guidelines for the diagnosis and treatment of osteoarthritis in China (2019 edition). Ann Translational Med. 2020;8(19):1213. doi:10.21037/atm-20-4665

46. McGrory B, Weber K, Lynott J, et al. The American Academy of Orthopaedic Surgeons Evidence-Based Clinical Practice Guideline on Surgical Management of Osteoarthritis of the Knee. The Journal of Bone and Joint Surgery. American Volume. 2016;98(8):688-692. doi:10.2106/JBJS.15.01311

47. Ruan A, Wang Q, Ma Y, et al. Efficacy and Mechanism of Electroacupuncture Treatment of Rabbits With Different Degrees of Knee Osteoarthritis: a Study Based on Synovial Innate Immune Response. Front Physiol. 2021;12:642178. doi:10.3389/ fphys. 2021.642178

48. Li B, Jing L, Jia L, et al. Acupuncture reduces pain in rats with osteoarthritis by inhibiting MCP2/CCR2 signaling pathway. Exp Biol Med. 2020;245(18):1722-1731. doi:10.1177/1535370220952342

49. Wu GW, Chen J, Huang YM, et al. Electroacupuncture Delays Cartilage Degeneration by Modulating Nuclear Factor- $\kappa B$ Signaling Pathway. Chin J Integr Med. 2019;25(9):677-683. doi:10.1007/ s11655-018-2916-8 
Journal of Pain Research

Dovepress

\section{Publish your work in this journal}

The Journal of Pain Research is an international, peer reviewed, open access, online journal that welcomes laboratory and clinical findings in the fields of pain research and the prevention and management of pain. Original research, reviews, symposium reports, hypothesis formation and commentaries are all considered for publication. The manuscript

Submit your manuscript here: https://www.dovepress.com/journal-of-pain-research-journa management system is completely online and includes a very quick and fair peer-review system, which is all easy to use. Visit http:// www.dovepress.com/testimonials.php to read real quotes from published authors. 